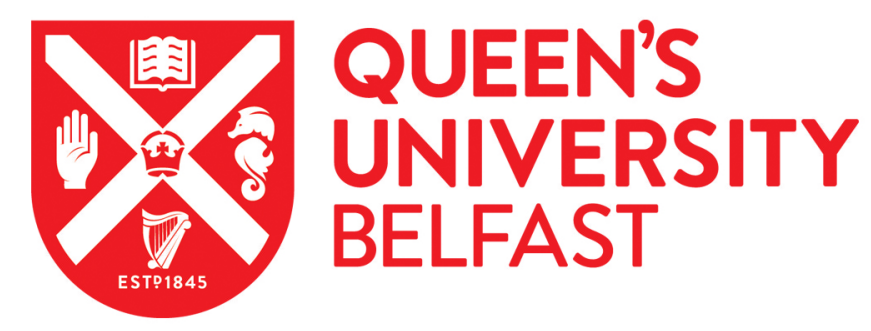

\title{
Yeast as a tool to express sugar acid transporters with biotechnological interest
}

Ribas, D., Sá-Pessoa, J., Soares-Silva, I., Paiva, S., Nygård, Y., Ruohonen, L., Penttilä, M., \& Casal, M. (2017). Yeast as a tool to express sugar acid transporters with biotechnological interest. FEMS Yeast Research. https://doi.org/10.1093/femsyr/fox005

\author{
Published in: \\ FEMS Yeast Research
}

Document Version:

Peer reviewed version

Queen's University Belfast - Research Portal:

Link to publication record in Queen's University Belfast Research Portal

Publisher rights

Copyright 2017 OUP

This work is made available online in accordance with the publisher's policies. Please refer to any applicable terms of use of the publisher.

\section{General rights}

Copyright for the publications made accessible via the Queen's University Belfast Research Portal is retained by the author(s) and / or other copyright owners and it is a condition of accessing these publications that users recognise and abide by the legal requirements associated with these rights.

Take down policy

The Research Portal is Queen's institutional repository that provides access to Queen's research output. Every effort has been made to ensure that content in the Research Portal does not infringe any person's rights, or applicable UK laws. If you discover content in the Research Portal that you believe breaches copyright or violates any law, please contact openaccess@qub.ac.uk. 


\title{
Yeast as a tool to express sugar acid transporters with biotechnological interest
}

David Ribas ${ }^{1}$, Joana Sá-Pessoa ${ }^{1}$, Isabel Soares-Silva ${ }^{1}$, Sandra Paiva ${ }^{1}$, Yvonne Nygård $^{2}$, Laura Ruohonen ${ }^{2}$, Merja Penttilä ${ }^{2}$, Margarida Casal ${ }^{1}$

${ }^{1}$ Centre of Molecular and Environmental Biology (CBMA), Department of Biology, University of Minho, Campus de Gualtar, Braga 4710-057, Portugal

${ }^{2}$ VTT Technical Research Centre of Finland Ltd, Tietotie 2, P.O. Box 1000, 02044 VTT Espoo, Finland

\author{
Corresponding authors: \\ Margarida Casal \\ Departamento de Biologia \\ Universidade do Minho \\ Campus de Gualtar \\ 4710-057 Braga \\ Portugal \\ Phone: +351253604044 \\ Fax: +351253678980 \\ email: mcasal@bio.uminho.pt,
}

\section{Keywords}

Sugar acids, Carboxylate transporters; Jen family; Yeast; microbial production of acids 


\section{Abstract}

Sugar acids can be used as platform chemicals to generate primary building blocks of industrially relevant products. Microbial production of these organic compounds at high yields requires the engineering of the enzymatic machinery and the presence of plasma membrane transporters able to export them outside the cells. In this study several yeast carboxylic acid transporters belonging to the Jen family were screened for the transport of biotechnologically relevant sugar acids, namely gluconic, saccharic, mucic, xylaric and xylonic acid, and functionally characterized in Saccharomyces cerevisiae. We show that Jen permeases are capable of transporting most of these sugar acids, although with different specificities. Saccharate is a substrate of the transporters ScJen1-S271Q and KIJen2, gluconate of CaJen2 and KIJen2, and xylarate and mucate of CaJen2. A molecular docking approach of these transporters identified the residues that play a major role in the substrate binding of these sugar acids, namely R188 (ScJen1), R122 (CaJen2) and R127 (KIJen2), all equivalent residues (TMS II). The identification of Jen members as sugar acid transporters can contribute to engineering efficient microbial cell factories with increased sugar acid production, as the ScJen1 is able to promote substrate efflux.

\section{Introduction}

About $50 \%$ of the most demanded biologically synthetized platform chemicals are organic acids, including sugar acids, such as gluconic, mucic, saccharic and xylonic acid (Mehtiö et al. 2016; Werpy and Petersen, 2004). The range of applications of these acids is very extended, embracing the food, pharmaceutical and chemical industry (Mehtiö et al. 2016).

The microbial production of carboxylic acids is an environmentally sound alternative to oil-based chemicals (Sauer et al. 2008). Escherichia coli and Saccharomyces cerevisiae have been suggested as promising cell factories to produce carboxylic acids, such as lactic, malic, xylonic and succinic acids (see review Alonso, Rendules and Diaz, 2015). Significant titres were obtained with these microbes (Liu and Jarboe, 2012; Toivari et al. 2012), although a major limiting factor to overcome during the fermentation process is the product toxicity (Holyoak et al. 1999; Piper et al. 1998; Piper et al. 2001; Nygård et al. 2014). Other organisms such as lactic-acid bacteria have been used for industrial purposes, although they have some well-known drawbacks: they have complex nutritional requirements due to their reduced ability to synthesize B-type vitamins and amino acids; they require costly downstream processing approaches and many bacterial species are unable to grow at low $\mathrm{pH}$ (Wee 
et al. 2005). The utilization of acid-tolerant microorganisms, such as the yeast $S$. cerevisiae with higher predisposition for growing at lower $\mathrm{pH}$ and enhanced resistance mechanisms to weak carboxylic acid stress, would reduce the cost for $\mathrm{pH}$ titrants (Leber and Da Silva, 2014). The toxic effect of an acid is intrinsically related to both the anion and proton accumulation in the cytoplasm, which affect the plasma membrane integrity (Lennen et al. 2011; Jarboe, Royce and Liu, 2013; Liu et al. 2013). The chemical state of the acid results from the intracellular $\mathrm{pH}$. When the extracellular $\mathrm{pH}$ is below the $\mathrm{p} K_{\mathrm{a}}$ of the acid, the undissociated/uncharged form of the molecule predominates, crossing the biological membranes by passive diffusion, depending on its lipid solubility and cell membrane permeability. However, when the $\mathrm{pH}$ is above the $\mathrm{p} K_{\mathrm{a}}$ of the carboxylic acid, the dissociated or anion form predominates, which implies a mediated transport mechanism to accomplish the uptake/export of the molecule (reviewed by Casal et al. 2008). In order to cope with high levels of carboxylic acid production, cell engineering approaches are required to introduce an efficient mechanism to transport the acid out of the cell, avoiding the intracellular accumulation of acid anions, and ultimately increasing the extracellular acid titres (Liu and Jarboe, 2012).

The Jen family, which belongs to the Major Facilitator Superfamily, is associated with plasma membrane transport of carboxylic acids in fungi. The first member of this family to be described was ScJen1 from $S$. cerevisiae, a lactate proton symporter with a $K_{m}$ of $0.29 \mathrm{mM}$ and a $V_{\max }$ of $0.4 \mathrm{nmol}^{-1} \mathrm{~s}^{-1} \mathrm{mg}^{-1}$ dry wt (Casal et al. 1999; Paiva et al. 2013). Besides lactate, ScJen1 also transports acetate, propionate and pyruvate (reviewed by Casal et al. 2016). The ScJen1 has 12 predicted transmembrane segments (TMS). Through rational mutagenesis of conserved amino acid residues ScJen1 structuralfunctional properties have been elucidated. The conserved motif ${ }^{379} \mathrm{NXX}[\mathrm{S} / \mathrm{T}] \mathrm{HX}[\mathrm{S} / \mathrm{T}] \mathrm{QD}{ }^{387}$, located towards the periplasmic side of the predicted TMS VII, is involved in the substrate translocation pathway (Soares-Silva et al. 2007). The N379 residue of this domain along with the N501 (TMS XI) and R188 (TMS II) residues were reported to be irreplaceable for the ScJen1 activity (Soares-Silva et al. 2007; Soares-Silva et al. 2011). Both $\mathrm{H} 383$ and D387 residues affect the specificity and transport capacity of the symporter. Additionally, the Q386 residue is involved in substrate affinity (Soares-Silva et al. 2007) and F270 (TMS V) and Q498 (TMS XI) are critical for substrate specificity, in particular for the differentiation between mono- and dicarboxylates (Soares-Silva et al. 2011).

Other Jen homologues from Candida albicans (Soares-Silva et al. 2004; Vieira et al. 2010), Kluyveromyces lactis (Lodi et al. 2004; Queirós et al. 2007), Debaryomyces hansenni (Soares-Silva et al. 2015), Yarrowia lipolytica (Dulermo et al. 2015; Guo et al. 
2015) and Aspergillus nidulans (Sá-Pessoa et al. 2015) were also reported to transport mono-, di- and/or tricarboxylates (reviewed in Casal et al. 2008, 2016). Ady2 is another carboxylate transporter from $S$. cerevisiae belonging to the acetate transporter family (AceTr) (Paiva et., 2004; Sá-Pessoa et al. 2013). Pacheco and colleagues (2012) in an attempt to create an improved $S$. cerevisiae strain for the production of lactate, overexpressed the ScJen1 and Ady2 transporters in a strain expressing the I-LDH gene from Lactobacillus casei, demonstrating their role as efflux permeases by successfully increasing the extracellular titration of lactate. The reversibility of ScJen1 transport had already been demonstrated in a previous work where the permease activity was reconstituted in membrane vesicles (Soares-Silva et al. 2003). Depending on the substrate and proton gradients, the ScJen1 was able to promote lactate uptake or efflux.

In this study we used the model organism S. cerevisiae jen1 $\Delta$ ady2 $\Delta$ (Soares-Silva et al. 2007) expressing distinct Jen homologues to assess their ability to transport gluconic, mucic, saccharic, xylonic and xylaric acid, an attractive C5 diacid despite not having biotechnological production. The identification of sugar acid transporters belonging to Jen family can be a valuable tool for the improvement of engineered yeasts strains tuned for the production of these acids.

\section{Materials and methods}

\section{Yeasts species, strains, plasmids and growth conditions}

The yeast strains and plasmids used in this work are listed in tables 1 and 2, respectively. The yeast cultures were maintained on slants of yeast extract $(1 \%, w / v)$, peptone $(1 \%, w / v)$, glucose $(2 \%, w / v)$ and agar $(2 \%, w / v)$ or in yeast nitrogen base (YNB medium, Difco), $0.67 \%$, w/v, supplemented with the adequate requirements for prototrophic growth. Yeast cells were grown in YNB medium. Carbon sources were glucose $(2 \%, \mathrm{w} / \mathrm{v})$, lactic $(0.5 \%, \mathrm{v} / \mathrm{v}, \mathrm{pH} 5.0)$ or succinic acid $(1 \%, \mathrm{w} / \mathrm{v}, \mathrm{pH} 5.0)$. Growth in liquid media was performed in Erlenmeyer flasks, with quintuple capacity of the volume of culture medium utilized. Cultures were always harvested during the exponential phase of growth. Cultures were incubated at proper temperature (as given in tables and legends) in an orbital incubator at $200 \mathrm{rpm}$. Growth evaluation in liquid medium was performed by OD measurement at $640 \mathrm{~nm}$. For derepression conditions glucose-grown cells were centrifuged, washed twice in sterile deionized water and cultivated in fresh YNB medium supplemented with lactic acid or succinic acid for $4 \mathrm{~h}$.

\section{Transport assays}


For uptake measurements cells were harvested during exponential phase by centrifugation (5000 rpm, 2 minutes). The samples were washed twice in ice-cold deionized water and resuspended in ice-cold deionized water to a final concentration of about 25-35 mg dry weight/mL. The reaction mixtures were prepared in $1.5 \mathrm{~mL}$ microtubes tubes containing $60 \mu \mathrm{L}$ of $\mathrm{KH}_{2} \mathrm{PO}_{4}(0.1 \mathrm{M}, \mathrm{pH} 5.0)$, and $30 \mu \mathrm{L}$ of the yeast cell suspension. After 2 minutes of incubation at $26^{\circ} \mathrm{C}$, the reaction was started by the addition of $10 \mu \mathrm{L}$ of a solution of radiolabelled substrate, at the desired $\mathrm{pH}$ and concentration, rapidly mixed by vortexing, and incubated at $26^{\circ} \mathrm{C}$. After one minute, $100 \mu \mathrm{L}$ of non-labelled substrate at $100 \mathrm{mM}$ was added, quickly mixed by vortexing and chilled on ice, to stop the reaction. The reaction solutions were centrifuged for 5 minutes at $13200 \mathrm{rpm}$. The supernatant was carefully rejected, the pellet was resuspended in $1 \mathrm{~mL}$ of deionized cold water and centrifuged for 5 minutes at 13200 rpm. The resulting pellet was resuspended in $1 \mathrm{~mL}$ of scintillation liquid (Opti-Phase HiSafe II; LKB FSA Laboratory Supplies). Non-specific ${ }^{14} \mathrm{C}$ adsorption to the cells, as well as the diffusion component, was determined by adding a 1000-fold concentrated mixture of labelled and unlabelled acid. The values estimated represent less than $5 \%$ of the total incorporated radioactivity. The inhibition assays were carried out by adding simultaneously the labelled and non-labelled substrates. The inhibition constant $\left(K_{\mathrm{i}}\right)$ was deduced by the effect of different concentrations of the non-labelled inhibitor in uptake velocities. The $K_{\mathrm{i}}$ values of competitive transport and the capacity to transport the inhibitory are an indirect indication for transport of the inhibitory molecule through the

carrier.

The following radioactive labelled substrates were utilized, D,L- $\left[{ }^{14} \mathrm{C}\right]$-lactic acid (Perkin Elmer) and $\left[{ }^{14} \mathrm{C}\right]$-succinic acid (Moravek Biochemicals) with a specific activity of 2000 dpm. Radioactivity was measured in a Packard Tri-Carb 2200 CA liquid scintillation spectrophotometer, with dpm correction.

The transport kinetics best fitting the experimental initial uptake rates, as well as statistical analysis (One-Way ANOVA) were determined by a computer-assisted nonlinear regression analysis using GraphPad Prism version 6.0 for Windows. The data shown are mean values of at least three independent experiments, with three replicas each.

\section{D structural modelling and molecular docking}

To obtain predicted Jen transporter 3D structures, the amino residues sequences were threaded through PDB library using LOMETS (Local Meta-Threading-Server) (Wu and Zhang, 2007). LOMETS (on-line web service for protein structure prediction) was used to generate a 3D model by collecting high-scoring target-to-template alignments from 
10 locally-installed threading programs (FFAS-3D, HHsearch, MUSTER, Neff-PPAS, pGenTHREADER, PPAS, PRC, PROSPECT2, SP3, and SPARKS-X). The threading folds were considered based on the "Confidence Score". The "Confidence Score" indicates the confidence of the predicted template which is based on a scoring function that takes into account the Z-score of the template, the confidence of the particular server and the sequence identity between the query and the template $\mathrm{Wu}$ and Zhang, 2007). The PipT phosphate transporter (PDB code 4J05A) was the top ranked template threading identified in LOMETS for ScJen1 and KIJen2, and the GLUT3 glucose transporter (PDB code 4zw9A) was the top ranked template threading identified in LOMETS for CaJen2.

Molecular docking simulations were performed using AutoDockvina with a Lamrkian genetic algorithm as scoring function (Trott and Olson, 2010). Chimera (Pettersen et al. 2004), a graphical user interface for AutoDock Vina, was used to perform virtual screening. The binding affinities and RMSD (root-mean-square deviation) scores for each ligand with nine different poses were determined. Primarily proteins and ligand molecules were prepared and optimized. These results were analysed with Chimera and the 2D and 3D ligand interaction images were rendered in Free Maestro version (Schrödinger Release 2016-1: Maestro, version 10.5, Schrödinger, LLC, New York, NY, 2016). The structure of all carboxylic acids was obtained from the Zinc database (Irwin and Shoichet, 2005). The docking studies were performed with the dissociated form of each carboxylic acid.

\section{Results}

\section{Screening ScJen1 alleles able to transport gluconic, mucic, saccharic, xylaric and xylonic acids}

The transport of radiolabelled lactic acid was assessed in the presence of gluconic,

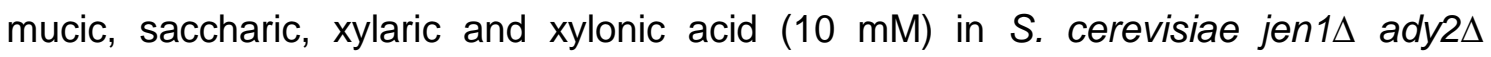
pScJen1. The presence of non-labelled acids allows the identification of potential inhibitors of lactic acid uptake, being indicative of interaction with the substrate binding site. Since no inhibition in lactate uptake was detected for the wild-type ScJen1 (data not shown), a collection of ScJen1 transporter alleles was tested. The alleles that possess altered kinetic parameters for carboxylic acid uptake were selected, namely A272G, Y284Q, Y284A, Q386A, Q498A, S271Q, F270G, F270A and F270Q/S271Q (Soares-Silva et. 2007; Soares-Silva et al. 2011). The ScJen1-S271Q was the only allele that presented a phenotype, where the uptake of labelled lactate was inhibited by $50 \%$ in the presence of non-labelled saccharic acid (Fig. 1A). A further characterization 
of the transport capacity of saccharate by the S271Q mutant was done by performing inhibitions of lactate uptake in the presence of this substrate at different concentrations. The Eadie-Hoffstee plots of the initial uptake rates of ${ }^{14} \mathrm{C}$-lactic acid uptake in the absence and presence of saccharic acid (20 and $30 \mathrm{mM}$ ) revealed a competitive inhibition profile for the S271Q mutant (Fig. 1B). These results suggest that saccharic acid is a substrate of the ScJen1S271Q transporter. The estimated $K_{i}$ value of saccharic acid was $21.8 \mathrm{mM}$ (Fig. 1B, inset).

Studies of modelling by docking of lactate and saccharate were performed for both ScJen1 and ScJen1-S271Q alleles (Fig. 2). The native ScJen1 structure displays two putative binding sites for lactate (Fig. 2A): one located close to the periplasmic side of the membrane; the other in the central pore closer to the cytoplasm. The docking of saccharate revealed a sole binding site in the ScJen1 structure, close to the periplasmic side of the membrane.

The predicted model of ScJen1-S271Q revealed the existence of two putative binding sites for both saccharate and lactate, suggesting that in this allele the two acids share the same binding sites (Fig. 2A). Taking into account the position of each ligand, as well as their structural overlap, it is noteworthy that the binding sites identified in Scjen1 and ScJen1-S271Q are occupying the same spatial position in the structure of the protein. A close view on the interactions between ligands and protein residues confirmed the previous observation (Fig. 2B). In the ScJen1 first binding site, saccharate interacts with the T382 and Q386 residues and lactate with the Q386 residue by hydrogen bonds. Moreover, in ScJen1 the second binding site exclusive for lactate displayed a hydrogen bond with the $\mathrm{Y} 497$ residue and a salt bridge with the R188 residue. Similarly, in the Scjen1-S271Q first binding site, saccharate interacts with the T382 residue and lactate with the Q386 residue. However, in the second binding site, saccharate interacts by a salt bridge with the $\mathrm{R} 188$ residue, which is also involved in lactate binding, as well as with the Y273, Y246 and N379 residues by hydrogens bonds. The residue Y497 is not involved in saccharate binding, but is important for lactate binding.

\section{Assessing non-saccharomyces yeast species ability to transport gluconic, mucic, saccharic, xylaric and xylonic acid}

Homologous proteins of Jen family, able to transport mono- (Jen1) and dicarboxylic acids (Jen2) were found in the yeasts Candida albicans (Silva et al. 2004; Vieira et al. 2010), Kluyveromyces lactis (Lodi et al. 2004; Queirós et al. 2007), Debaryomyces hansenni (Soares-Silva et al. 2015) and Yarrowia lipolytica (Dulermo et al. 2015; Guo et al. 2015). The uptake of labelled lactic and succinic acid was measured in the above 
mentioned yeasts species, in the presence of gluconic, mucic, saccharic, xylaric and xylonic acid (Fig. 3). In all these species the uptake of labelled lactic acid was not inhibited by the presence of any of the acids tested. Similar results were found for the uptake of labelled succinic acid in D. hansenii and $Y$. lipolytica. However, in C. albicans and $K$. lactis the uptake of labelled succinic acid was inhibited in the presence of all the above mentioned acids. In addition, in $D$. hansenii cells lactate and succinate uptake was higher in the presence of xylonic and xylaric acid, as well as mucic, saccharic and xylonic acid, respectively. Whereas in $K$. lactis, the lactate uptake increased in the presence of mucic and and xylonic acid.

\section{Specificity profiles of CaJen1 and CaJen2 for gluconic, mucic, saccharic, xylaric and xylonic acid}

S. cerevisiae jen1 $\Delta$ ady2 $\Delta$ strains expressing the monocarboxylate CaJen1 or the dicarboxylate CaJen2 homologues were used to evaluate the inhibition profile of gluconic, mucic, saccharic, xylaric and xylonic acid $(10 \mathrm{mM})$. None of these acids inhibited the uptake of ${ }^{14} \mathrm{C}$-lactic acid uptake in cells expressing CaJen1 (Fig. 4A). In cells expressing CaJen2, succinate uptake was inhibited by $50 \%, 40 \%$ and $80 \%$ by mucic, xylaric and gluconic acid, respectively (Fig. 4B).

Eadie-Hofstee plots of the initial uptake rates of labelled succinic acid in the presence of mucic, xylaric and gluconic acid revealed a competitive inhibition profile for these acids (Fig. 5). The inhibition constants for xylaric acid ( $\left.K_{i} 20.2 \mathrm{mM}\right)$ and gluconic acid $\left(K_{i} 25.9 \mathrm{mM}\right)$ were estimated (Fig. 5, insets). Mucic acid acted as an inhibitor when using the highest concentration possible to use in solution $(20 \mathrm{mM})$, impairing the estimation of the $K_{i}$ for this acid (Fig. 5C).

Molecular docking of CaJen2 with malate, succinate, gluconate, mucate or xylarate unveiled one single putative binding site common to all these ligands (Fig. 6A). Moreover, the R122 residue interacts with all the substrates analysed, either by hydrogen bonds or by salt bridges (Fig. 6B). Interestingly, mucate interacted with the same residues (W83, R122 and Q204) as malate and succinate, whereas gluconate interacted with Q204 like the aforementioned ligands and additionally with Y417. Xylarate only interacted directly with R122.

\section{Specificity profiles of KIJen1 and KLJen2 for gluconic, mucic, saccharic, xylaric and xylonic acid}

S. cerevisiae jen $1 \Delta$ ady2 $\Delta$ cells expressing the $K$. lactis monocarboxylic KIJen1 and dicarboxylic KIJen2 transporters (Lodi et al.2004; Queirós et al. 2007) were used to assess their specificity for gluconic, mucic, saccharic, xylaric and xylonic acids. The 
uptake of ${ }^{14} \mathrm{C}$-lactic acid in cells expressing the KIJen 1 was not inhibited by any of the carboxylic acids mentioned above (Fig. 7A). Nonetheless, both saccharic and gluconic acid (10 mM) significantly decreased the uptake of ${ }^{14} \mathrm{C}$-succinic acid in cells expressing KIJen2 (Fig. 7B).

Eadie-Hoffstee plots of the initial uptake rates of ${ }^{14} \mathrm{C}$-succinic acid uptake in the presence of different concentrations of saccharic and gluconic acids displayed a competitive inhibition profile (Fig. 8). The $K_{i}$ value of gluconic acid was $22.7 \mathrm{mM}$ (Fig. $8 \mathrm{~A}$, inset) and the $K_{i}$ of saccharic acid was $24.8 \mathrm{mM}$ (Fig. 8B, inset).

The docking of malate, succinate, gluconate, or saccharate in KIJen2 allowed to uncover a single putative binding site common to all the ligands analysed (Fig. 9A). The R127 residue is involved in direct interactions with all the carboxylic acids tested, predominantly by salt bridges (Fig. 9B). This residue was the only residue interacting with malate, succinate and gluconate. Saccharate additionally interacted with $\mathrm{H} 308$.

\section{Discussion}

This study focused on the identification of yeast carboxylate transporters belonging to Jen family with specificity for sugar acids with biotechnological interest. Ultimately these transporters could be used as a tool to improve production titres in engineered strains of $S$. cerevisiae tuned for the production of sugar acids. Our results show that saccharate is a substrate of the transporters ScJen1-S271Q and KIJen2, gluconate of CaJen2 and KIJen2, and xylarate and mucate of CaJen2.

The expression system used in this work has been successfully utilized before for the characterization of the Jen homologues of K. lactis (Queirós, et al. 2007), C. albicans (Soares-Silva, et al. 2004, Vieira, et al. 2010), D. hansenii (Soares-Silva et al. 2015)

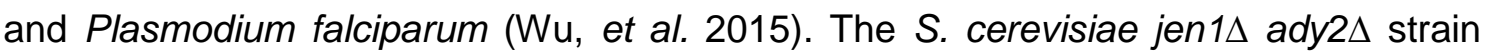
(Soares-Silva et al. 2011), which presents no activity for the uptake of carboxylates, was used to express the Jen permeases from C. albicans, and $K$. lactis as well as the ScJen1 mutant alleles.

Herein, we describe that $S 271 Q$ allele shows a $K_{i}$ value of $21.8 \mathrm{mM}$ for saccharate. The S271Q allele was previously associated to loss of function for selenite transport, but not for lactate, uncovering the role of this residue in the ScJen1 transporter specificity. The gain of function of ScJen1 enabling the transport of dicarboxylic acids in mutant alleles of ScJen1 was already reported (Soares-Silva et al. 2011). The F270G, F270G/S271Q and Q498A mutants are able to transport the dicarboxylates succinic and malic acid with a $K_{i}$ of $1.9 \mathrm{mM}$ and $3.4 \mathrm{mM}$ (F270G), $1.9 \mathrm{mM}$ and $28.1 \mathrm{mM}$ (F270G/S271Q) and $29.8 \mathrm{mM}$ and $33.5 \mathrm{mM}$ (Q498A), respectively (Soares-Silva et al. 2011). Molecular docking analyses in the present study showed that S271Q mutation 
plays a role in saccharate transport, although it never interacts directly with this substrate. This phenotype could be due to the localization of S271 residue in the vicinity of F270, a critical residue in substrate specificity that has a significant impact in ScJen1 transport characteristics. While ScJen1 allele only displays one putative binding site common to saccharate and lactate in the upper part of the pore, the ScJen1-S271Q mutant has an extra putative binding site for saccharate shared with lactate, right in the middle of the pore. This last binding site comprises interactions between saccharate and the protein, involving two of the most important residues of ScJen1 functionality, the R188 and N379 residues. Previous docking analyses have reported that the $\mathrm{R} 188$ residue plays a major dynamic role mediating the orderly relocation of the substrate (Soares-Silva et al. 2011). This interaction between saccharate and both the R188 and N379 residues, observed exclusively in the S271Q mutant, could explain the reason why lactate uptake was reduced in the presence of saccharate, i.e. saccharate is competing with lactate for the transporter binding site.

It was previously reported that Jen 1 family has members in several yeasts, including $C$. albicans, D. hansenii, K. lactis and Y. lipolytica. In order to identify the possible involvement of Jen transporters in the transport of sugar acids, the uptake of lactate and succinate was tested in these yeasts in the presence of sugar acids. However, only the uptake of radiolabelled succinic acid was inhibited by gluconic, mucic, saccharic, xylaric or xylonic acid in cells of $C$. albicans and $K$. lactis. Interestingly the uptake of lactate and succinate was higher in $D$. hansenii in the presence of some of the sugar acids tested. Although to a lesser extent, a similar profile was observed in $K$. lactis cells. It is likely that in these yeast species, the presence of these acids triggers a stimulation of lactate and succinate uptake by unknown mechanisms.

The inhibitory effect of sugar acids in the radiolabelled succinic acid uptake was only detected in S. cerevisiae jen $1 \Delta$ ady2 $\Delta$ transformed with the dicarboxylate transporters, CaJen2 and KIJen2. Molecular docking experiments with carboxylic acids shown to inhibit the transport of succinate were carried out. Malate was also included in the docking tests, since it is a known substrate for CaJen2 and KIJen2 (Lodi et al. 2004; Queirós et al. 2007; Soares-Silva et al. 2004; Vieira et al. 2010). In both CaJen2 and KIJen2 docking experiments only one putative binding site common to all carboxylic acids tested was found. In CaJen2, mucate presented a similar binding pattern as the known transported substrates (i.e. malate and succinate, interacting with the same residues (W83, R122, and Q204), whereas gluconate interacted with Q204, R122 and Y417 and xylarate bound exclusive to R122. Interestingly, the Q204 residue is the equivalent of the F270 residue from ScJen1, which was described as critical for substrate specificity (Soares-Silva et al. 2011). Actually, the F270Q mutation in ScJen1 
increased the range of substrate spectrum and it is likely to be one of the reasons for CaJen2 transporting such a number of carboxylic acids and particularly dicarboxylic acids. On the other hand, in KlJen2 docking tests, gluconate only interacted with the $\mathrm{R} 127$ residue in the same way as malate and succinate, whereas saccharate, besides R127 interaction, also interacted with $\mathrm{H} 308$. The $\mathrm{H} 308$ residue is the equivalent of H383 residue in ScJen1, which was described to be involved in substrate specificity and transport capacity (Soares-Silva et al. 2011). Similarly to ScJen1, there is a critical residue that seems to play an important role during substrate binding in CaJen2 and KIJen2. This residue is R122 (CaJen2) and R127 (KIJen2), respectively. These residues are the equivalent residues of R188 from ScJen1, known to be irreplaceable for transport activity (Soares-Silva et al. 2011).

Herein we found strong evidences that saccharate is a substrate of the transporters ScJen1-S271Q and KIJen2, gluconate of CaJen2 and KIJen2, and xylarate and mucate of CaJen2. Considering the nature of sugar acids, it is likely that the transport of these molecules is by an active mechanism, similarly to mono- and dicarboxylates (Casal et al. 1999; Soares-Silva et al. 2004; Queirós et al. 2007). Moreover, we did not find any Jen transporter with specificity for xylonate although succinate transport in the yeasts $C$. albicans and $K$. lactis was inhibited by this acid, suggesting the presence of an alternative transport system for succinate that binds xylonate. According to the transport classification database (TCDB; http://www.tcdb.org) there are few characterized eukaryotic transporters with specificity for the sugar acids here tested. Two gluconate transporters are identified, the Ght3 gluconate: $\mathrm{H}^{+}$symporter from Schizosaccharomyces pombe and the CIC-5 of Sus scrofa, a $\mathrm{Cl}^{-}: \mathrm{H}^{+}$antiporter with substrate preference for acetate and gluconate (Heiland et al. 2000; Scheel et al. 2005). The Homo sapiens CIC5 homologue (CIC-5) is associated with Dent's disease (Stechman, Loh and Thakker 2007); the SLC26A7 of $H$. sapiens is a chloride/sulfate/oxalate permease or channel, with specificity for saccharate (Kim et al. 2005); there are no eukaryotic transporters known for xylonate, xylarate and mucate, and only one prokaryotic putative mucate: $\mathrm{H}^{+}$symporter is reported, the GarP of $E$. coli (Moraes and Reithmeier, 2012).

The results of this study have high biotechnological potential for the microbial production of sugar acids with added industrial value. The transporter proteins here identified could be (over)expressed in industrial production organisms, where acid accumulation and tolerance is a bottleneck. The ScJen1 permease and other Jen1 homologues transport their substrates by a proton symport mechanism, and similarly to other permeases it is possible to reverse the uptake activity if the substrate gradient and/or proton motive force favours this mechanism (Casal et al. 1999; Soares-Silva et 
al. 2003; Soares-Silva et al. 2004; Queirós et al. 2007). In reconstituted heterologous $P$. pastoris membrane vesicles, it was demonstrated that ScJen1 is able to export lactic acid after a proton gradient change or the addition of another substrate of the permease, like pyruvate, at high concentrations (Soares-Silva et al. 2003). Considering the role of ScJen1 in lactate efflux (Pacheco et al. 2012), it is expectable that the expression of Jen permeases in sugar acid producing strains will increase the flux towards extracellular product accumulation. Mojzita et al. (2010) suggested that export of mucate may be limiting in Hypocrea jecorina. Kell et al. (2015) argued that transporters are largely undervalued proteins that could greatly contribute to the control of biotechnological fluxes. In addition to gain of functions obtained via random mutagenizes, several studies already demonstrate rational design approaches for improved transporters: beyond the studies of Soares-Silva and colleagues (2008 and 2011) previously pointed here and showing the effect of site-directed mutagenesis in the transport affinity and specificity of ScJen1; MdfA of E. coli has been converted from a mono into a divalent cationic drug transporter by inserting an additional acidic residue into the putative recognition pocket (Tirosh et al. 2012); there are also various both rational and site-directed mutagenesis attempts on engineering xylose transport and thus improving xylose fermentation in yeast (Young et al. 2014, Farwick et al. 2014).

Increased knowledge on transporters and their specificity is needed for predictive and quantitative structure-activity relationship models, ultimately leading to design of transporters (Kell et al. 2015). The docking studies of the acids performed in this study provide leads on how to further engineer these transporters or their homologues for altered affinity and specificity. In conclusion, this work provides novel understanding on sugar acids transport and is a step forward in developing microbial production of sugar acids with added industrial value.

\section{Funding}

This work was supported by: the strategic programme UID/BIA/04050/2013 (POCl-010145-FEDER-007569) and the project PTDC/BIAMIC/5184/2014 funded by national funds through the FCT I.P. and by the ERDF through the COMPETE 2020 - Programa Operacional Competitividade e Internacionalização (POCI); by the project EcoAgriFood: Innovative green products and processes to promote AgriFood BioEconomyl" (operação NORTE-01-0145-FEDER-000009), supported by Norte Portugal Regional Operational Programme (NORTE 2020), under the PORTUGAL 2020 Partnership Agreement, through the European Regional Development Fund (ERDF). DR acknowledges FCT for the SFRH/BD/96166/2013 PhD grant. 


\section{References}

Alonso S, Rendueles M, Díaz M. Microbial production of specialty organic acids from renewable and waste materials. Critical Reviews in Biotechnology 2015;35: 497-513.

Casal M, Paiva S, Andrade RP et al. The lactate-proton symport of Saccharomyces cerevisiae is encoded by JEN1. J Bacteriol 1999;181: 2620-3.

Casal M, Paiva S, Queiros O et al. Transport of carboxylic acids in yeasts. FEMS Microbiol Rev 2008;32: 974-94.

Casal M, Queiros O, Talaia G et al. Carboxylic Acids Plasma Membrane Transporters in Saccharomyces cerevisiae. Adv Exp Med Biol 2016;892: 229-51.

Dulermo R, Gamboa-Melendez H, Michely S et al. The evolution of Jen3 proteins and their role in dicarboxylic acid transport in Yarrowia. Microbiologyopen 2015;4: 100-20.

Farwick A, Bruder S, Schadeweg V et al. Engineering of yeast hexose transporters to transport d-xylose without inhibition by d-glucose. Proc Natl Acad Sci U S A 2014;111: 5159-64.

Guo H, Liu P, Madzak C et al. Identification and application of keto acids transporters in Yarrowia lipolytica. Sci Rep 2015;5.

Heiland S, Radovanovic N, Höfer $M$ et al. Multiple Hexose Transporters of Schizosaccharomyces pombe. Journal of Bacteriology 2000;182: 2153-62.

Holyoak CD, Bracey D, Piper PW et al. The Saccharomyces cerevisiae weak-acidinducible $A B C$ transporter Pdr12 transports fluorescein and preservative anions from the cytosol by an energy-dependent mechanism. J Bacteriol 1999;181: 4644-52.

Irwin JJ, Shoichet BK. ZINC--a free database of commercially available compounds for virtual screening. J Chem Inf Model 2005;45: 177-82.

Jarboe LR, Royce LA, Liu P. Understanding biocatalyst inhibition by carboxylic acids. Front Microbiol 2013;4: 272.

Kell DB, Swainston N, Pir P et al. Membrane transporter engineering in industrial biotechnology and whole cell biocatalysis. Trends in Biotechnology 2015;33: 237-46.

Kim KH, Shcheynikov N, Wang Y et al. SLC26A7 Is a Cl- Channel Regulated by Intracellular pH. Journal of Biological Chemistry 2005;280: 6463-70.

Leber C, Da Silva NA. Engineering of Saccharomyces cerevisiae for the synthesis of short chain fatty acids. Biotechnol Bioeng 2014;111: 347-58.

Lennen RM, Kruziki MA, Kumar K et al. Membrane stresses induced by overproduction of free fatty acids in Escherichia coli. Appl Environ Microbiol 2011;77: 8114-28.

Liu P, Chernyshov A, Najdi T et al. Membrane stress caused by octanoic acid in Saccharomyces cerevisiae. Appl Microbiol Biotechnol 2013;97: 3239-51.

Liu P, Jarboe LR. Metabolic engineering of biocatalysts for carboxylic acids production. Comput Struct Biotechnol J 2012;3: e201210011.

Lodi T, Fontanesi F, Ferrero I et al. Carboxylic acids permeases in yeast: two genes in Kluyveromyces lactis. Gene 2004;339: 111-9.

Mehtiö T, Toivari M, Wiebe MG et al. Production and applications of carbohydratederived sugar acids as generic biobased chemicals. Critical Reviews in Biotechnology 2016;36: 904-16.

Mojzita D, Wiebe M, Hilditch $S$ et al. Metabolic Engineering of Fungal Strains for Conversion of d-Galacturonate to meso-Galactarate. Appl Environ Microbiol 2010;76: 169-75.

Moraes TF, Reithmeier RAF. Membrane transport metabolons. Biochimica et Biophysica Acta (BBA) - Biomembranes 2012;1818: 2687-706.

Nygård $Y$, Mojzita $D$, Toivari $M$ et al. The diverse role of Pdr12 in resistance to weak organic acids. Yeast 2014;31: 219-32.

Pacheco A, Talaia G, Sa-Pessoa J et al. Lactic acid production in Saccharomyces cerevisiae is modulated by expression of the monocarboxylate transporters Jen1 and Ady2. FEMS Yeast Res 2012;12: 375-81. 
Paiva S, Devaux F, Barbosa $S$ et al. Ady2p is essential for the acetate permease activity in the yeast Saccharomyces cerevisiae. Yeast 2004;21: 201-10.

Paiva S, Strachotova D, Kucerova $\mathrm{H}$ et al. The transport of carboxylic acids and important role of the Jen $1 p$ transporter during the development of yeast colonies. Biochem J 2013;454: 551-8.

Pettersen EF, Goddard TD, Huang CC et al. UCSF Chimera--a visualization system for exploratory research and analysis. J Comput Chem 2004;25: 1605-12.

Piper $\mathrm{P}$, Calderon $\mathrm{CO}$, Hatzixanthis $\mathrm{K}$ et al. Weak acid adaptation: the stress response that confers yeasts with resistance to organic acid food preservatives. Microbiology 2001;147: 2635-42.

Piper $\mathrm{P}$, Mahe $\mathrm{Y}$, Thompson $\mathrm{S}$ et al. The pdr12 $\mathrm{ABC}$ transporter is required for the development of weak organic acid resistance in yeast. EMBO J 1998;17: 4257-65.

Queirós O, Pereira L, Paiva S et al. Functional analysis of Kluyveromyces lactis carboxylic acids permeases: heterologous expression of KIJEN1 and KIJEN2 genes. Curr Genet 2007;51: 161-9.

Sá-Pessoa J, Amillis S, Casal M et al. Expression and specificity profile of the major acetate transporter AcpA in Aspergillus nidulans. Fungal Genetics and Biology 2015;76: 93-103.

Sá-Pessoa J, Paiva S, Ribas D et al. SATP (YaaH), a succinate-acetate transporter protein in Escherichia coli. Biochem J 2013;454: 585-95.

Sauer M, Porro D, Mattanovich D et al. Microbial production of organic acids: expanding the markets. Trends in Biotechnology 2008;26: 100-8.

Scheel O, Zdebik AA, Lourdel S et al. Voltage-dependent electrogenic chloride/proton exchange by endosomal CLC proteins. Nature 2005;436: 424-7.

Soares-Silva I, Paiva S, Diallinas $G$ et al. The conserved sequence NXX[S/T]HX[S/T]QDXXXT of the lactate/pyruvate:H(+) symporter subfamily defines the function of the substrate translocation pathway. Mol Membr Biol 2007;24: 464-74.

Soares-Silva I, Paiva S, Kotter P et al. The disruption of JEN1 from Candida albicans impairs the transport of lactate. Mol Membr Biol 2004;21: 403-11.

Soares-Silva I, Ribas D, Foskolou IP et al. The Debaryomyces hansenii carboxylate transporters Jen1 homologues are functional in Saccharomyces cerevisiae. FEMS Yeast Res 2015;15.

Soares-Silva I, Sa-Pessoa J, Myrianthopoulos V et al. A substrate translocation trajectory in a cytoplasm-facing topological model of the monocarboxylate/ $\mathrm{H}(+)$ symporter Jen1p. Mol Microbiol 2011;81: 805-17.

Soares-Silva I, Schuller D, Andrade RP et al. Functional expression of the lactate permease Jen1p of Saccharomyces cerevisiae in Pichia pastoris. Biochem J 2003;376: 781-7.

Stechman MJ, Loh NY, Thakker RV. Genetics of Hypercalciuric Nephrolithiasis. Annals of the New York Academy of Sciences 2007;1116: 461-84.

Tirosh O, Sigal N, Gelman A et al. Manipulating the drug/proton antiport stoichiometry of the secondary multidrug transporter MdfA. Proceedings of the National Academy of Sciences 2012;109: 12473-8.

Toivari MH, Nygard Y, Penttila M et al. Microbial D-xylonate production. Appl Microbiol Biotechnol 2012;96: 1-8.

Trott O, Olson AJ. AutoDock Vina: improving the speed and accuracy of docking with a new scoring function, efficient optimization, and multithreading. $J$ Comput Chem 2010;31: 455-61.

Vieira N, Casal M, Johansson B et al. Functional specialization and differential regulation of short-chain carboxylic acid transporters in the pathogen Candida albicans. Mol Microbiol 2010;75: 1337-54.

Wee YJ, Yun JS, Lee YY et al. Recovery of lactic acid by repeated batch electrodialysis and lactic acid production using electrodialysis wastewater. $J$ Biosci Bioeng 2005;99: 104-8. 
Werpy T, Petersen G. Top Value Added Chemicals from Biomass: Volume I -- Results of Screening for Potential Candidates from Sugars and Synthesis Gas, DOI 10.2172/15008859. United States, 2004.

Wu B, Rambow J, Bock $S$ et al. Identity of a Plasmodium lactate/H+ symporter structurally unrelated to human transporters. Nature Communications 2015;6: 6284.

Wu S, Zhang Y. LOMETS: a local meta-threading-server for protein structure prediction. Nucleic Acids Res 2007;35: 3375-82.

Young EM, Tong A, Bui $\mathrm{H}$ et al. Rewiring yeast sugar transporter preference through modifying a conserved protein motif. Proc Natl Acad Sci U S A 2014;111: 131-6.

A

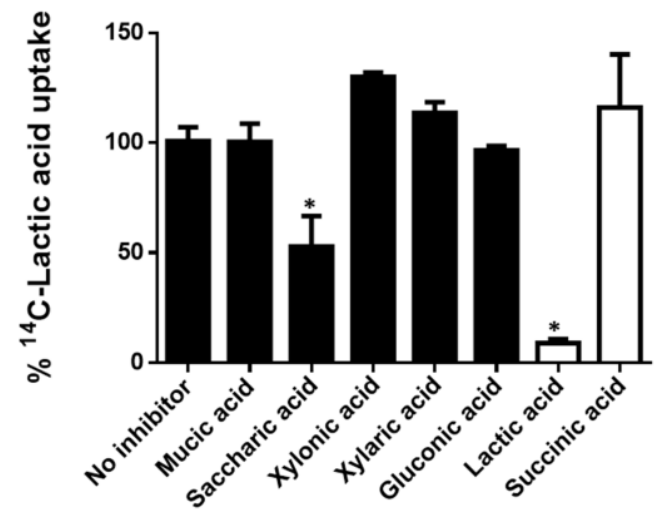

B

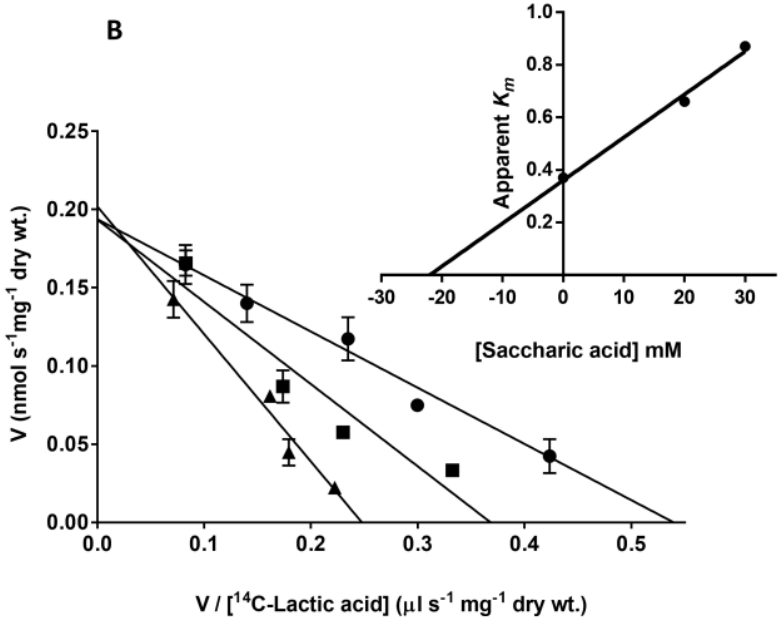

Fig.1

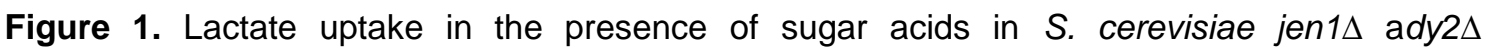
expressing the ScJen1-S271Q. A) Relative capacity (\%) of ${ }^{14} \mathrm{C}$-lactic acid uptake $(60 \mu \mathrm{M})$, at $\mathrm{pH}$ $5.0,26^{\circ} \mathrm{C}$, in the absence or presence of non-labelled sugar acids (10 mM). Lactic acid was used as positive control and succinic acid as negative control. B) Eadie-Hoffstee plots of the initial uptake rates of ${ }^{14} \mathrm{C}$-lactic acid as a function of the acid concentration at $\mathrm{pH} 5.0,26^{\circ} \mathrm{C}$, in the absence $(\bullet)$ and in the presence of saccharic acid $20 \mathrm{mM}(\boldsymbol{\square})$ and $30 \mathrm{mM}(\boldsymbol{\Delta})$. Inset: the apparent $K_{m}$ of labelled lactic acid uptake plotted against the saccharic acid concentration. The estimated $K_{i}$ is $21.8 \mathrm{mM}$ for total saccharic acid. The data shown are mean values of at least three independent experiments and the error bars represent the standard deviation. *Significant differences $(p<0.05)$. 


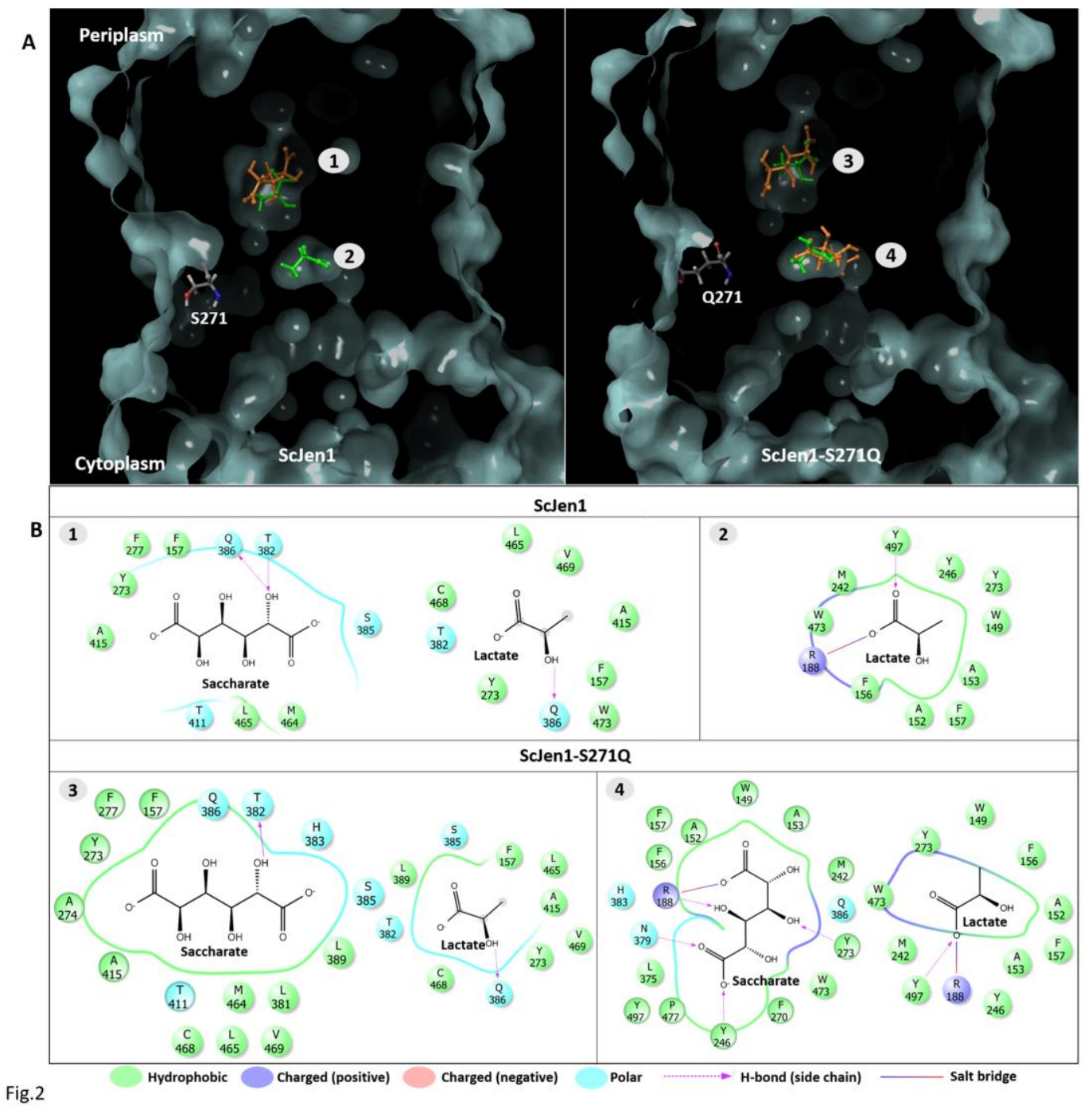

Figure 2. Molecular docking of ScJen1 and ScJen1-S271Q with saccharate (orange) and lactate (green). A) The 3D transversal view of ScJen1 and ScJen1-S271Q protein surface with identification of the lactate and saccharate binding sites $(1,2,3,4)$ obtained in the docking analysis. The 3D ScJen1 and ScJen1-S271Q structural models used in this study were obtained from the crystal structure of PiPT transporter (PDB 4J05). B) 2D view of the molecular structure of the interaction between the ligands (saccharate and lactate) and the proteins (ScJen1 and ScJen1-S271Q). 1, 2, 3 and 4 are relative to the binding sites identified. The arrows indicate hydrogen bond interaction between the amino acid residues and functional groups or atoms in the molecular structure of the ligand. 

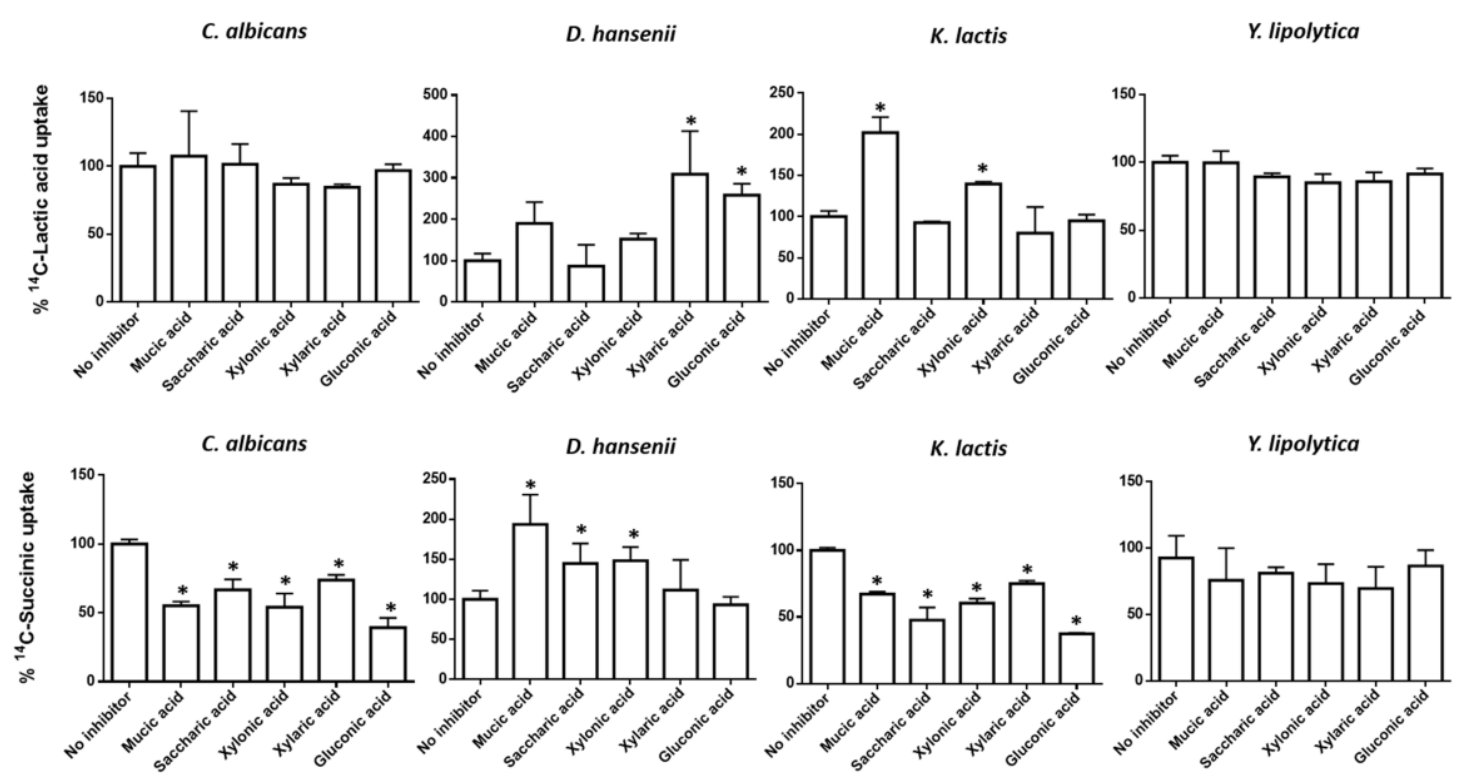

Fig.3

Figure 3. Relative capacity (\%) of lactate and succinate uptake in $C$. albicans, $D$. hansenii, $K$. lactis and $Y$. lipolytica measured in the absence and presence of non-labelled mucic, saccharic, xylonic, xylaric and gluconic acid $(10 \mathrm{mM}), \mathrm{pH} 5.0$ and $26^{\circ} \mathrm{C}$. The data shown are mean values of at least three independent experiments and the error bars represent the standard deviation. *Significant differences $(p \leq 0.05)$.

A

S. cerevisiae jen $1 \Delta$ ady2 $\Delta$ pCaJen1

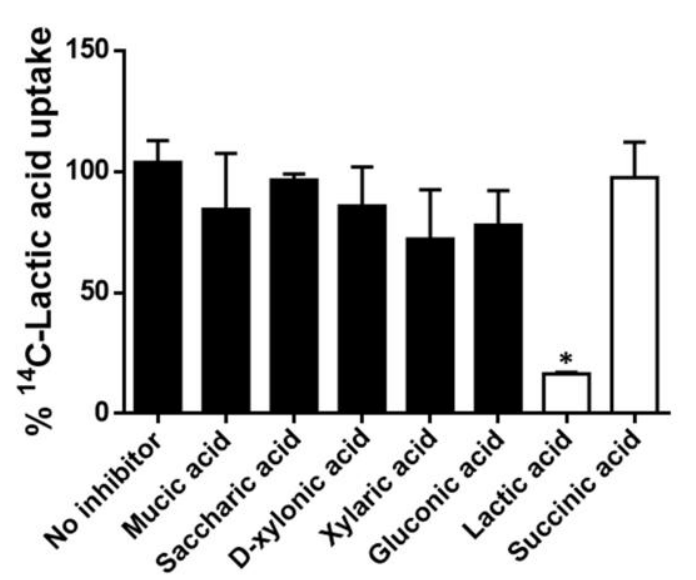

B

S. cerevisiae jen $1 \Delta$ ady $2 \Delta$ pCaJen2

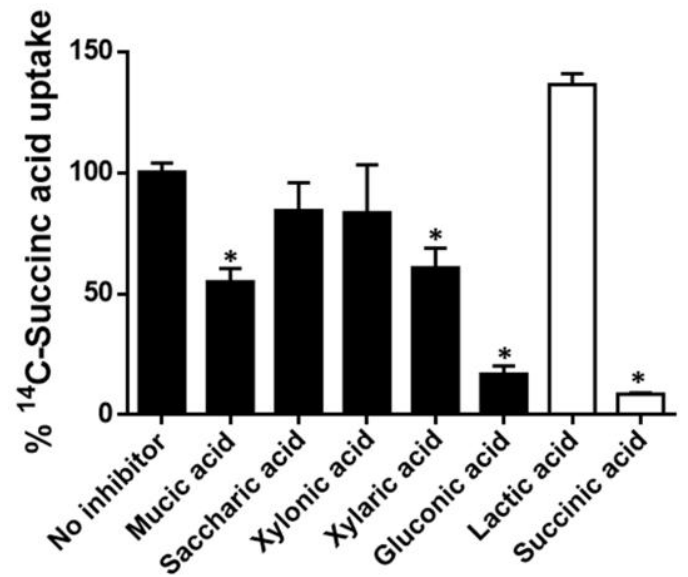

Fig. 4

Figure 4. Relative capacity (\%) of S. cerevisiae jen $1 \Delta$ ady2 $2 \Delta$ cells expressing C. albicans homologues (CaJen1 and CaJen2) to transport lactate and succinate in the absence and presence of mucic, saccharic, xylonic, xylaric and gluconic acid. A) Uptake of ${ }^{14} \mathrm{C}$-lactic acid $(60$ $\mu \mathrm{M})$ in cells transformed with pCaJen1 plasmid. B) Uptake of ${ }^{14} \mathrm{C}$-succinic acid $(20 \mu \mathrm{M})$ in cells transformed with pCaJen2. The data shown are mean values of at least two independent experiments and the error bars represent the standard deviation. ${ }^{*}$ Significant differences $(p \leq$ 0.05). 

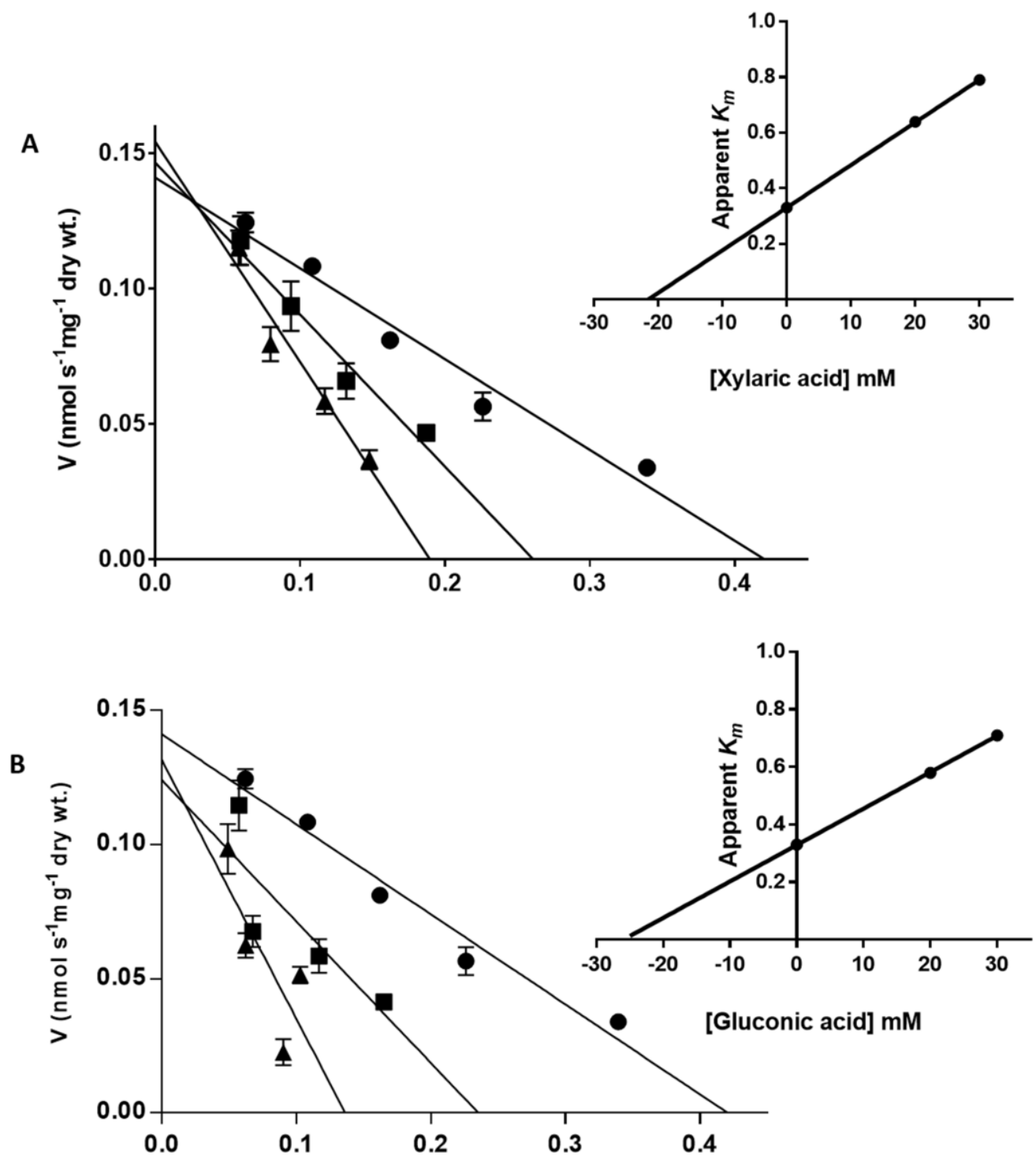

C

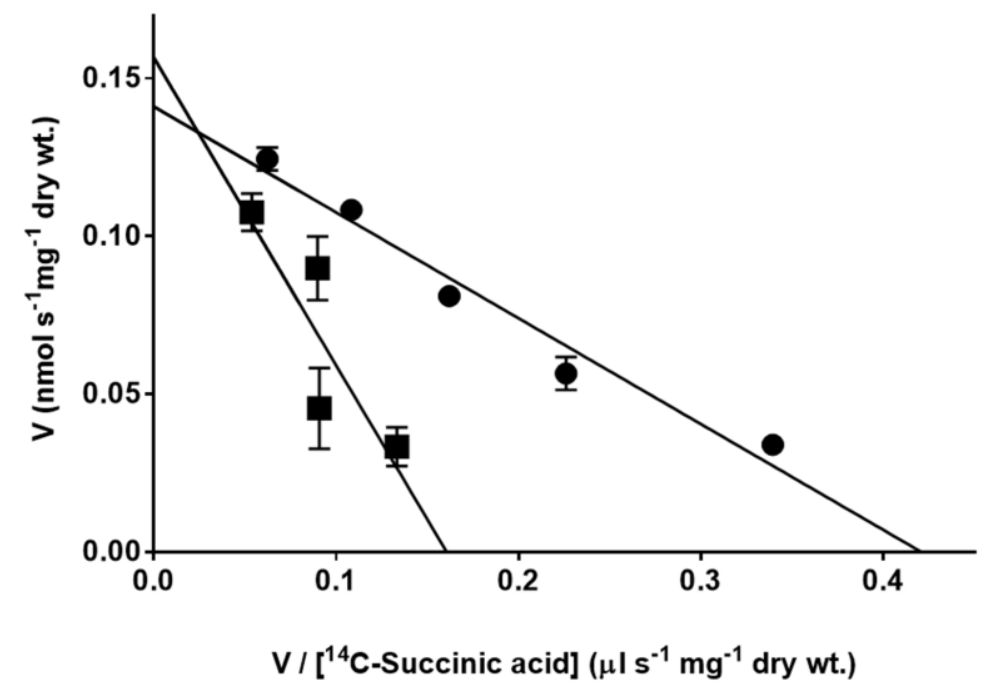

Fig.5 
Figure 5. Eadie-Hoffstee plots of the initial uptake rates of ${ }^{14} \mathrm{C}$-succinic acid as a function of the acid concentration at $\mathrm{pH} 5.0,26^{\circ} \mathrm{C}$ in S. cerevisiae jen $1 \Delta$ ady $2 \Delta$ pCaJen2 in the presence of:

A) no inhibitor $(\bullet)$, xylaric acid $20 \mathrm{mM}(\boldsymbol{\bullet})$ and xylaric acid $30 \mathrm{mM}(\mathbf{\Delta})$;

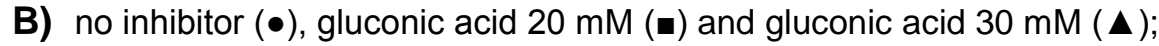

C) no inhibitor $(\bullet)$, mucic acid $20 \mathrm{mM}(\mathbf{\bullet})$.

Insets: the apparent $K_{m}$ of labelled succinic acid uptake was plotted against the concentration of xylaric (A) and gluconic (B) acid. The estimated $K_{i}$ is $20.2 \mathrm{mM}$ for total xylaric acid and and 25.9 $\mathrm{mM}$ for total gluconic acid. For mucic acid it was not possible to estimate the $K_{i}$ value.

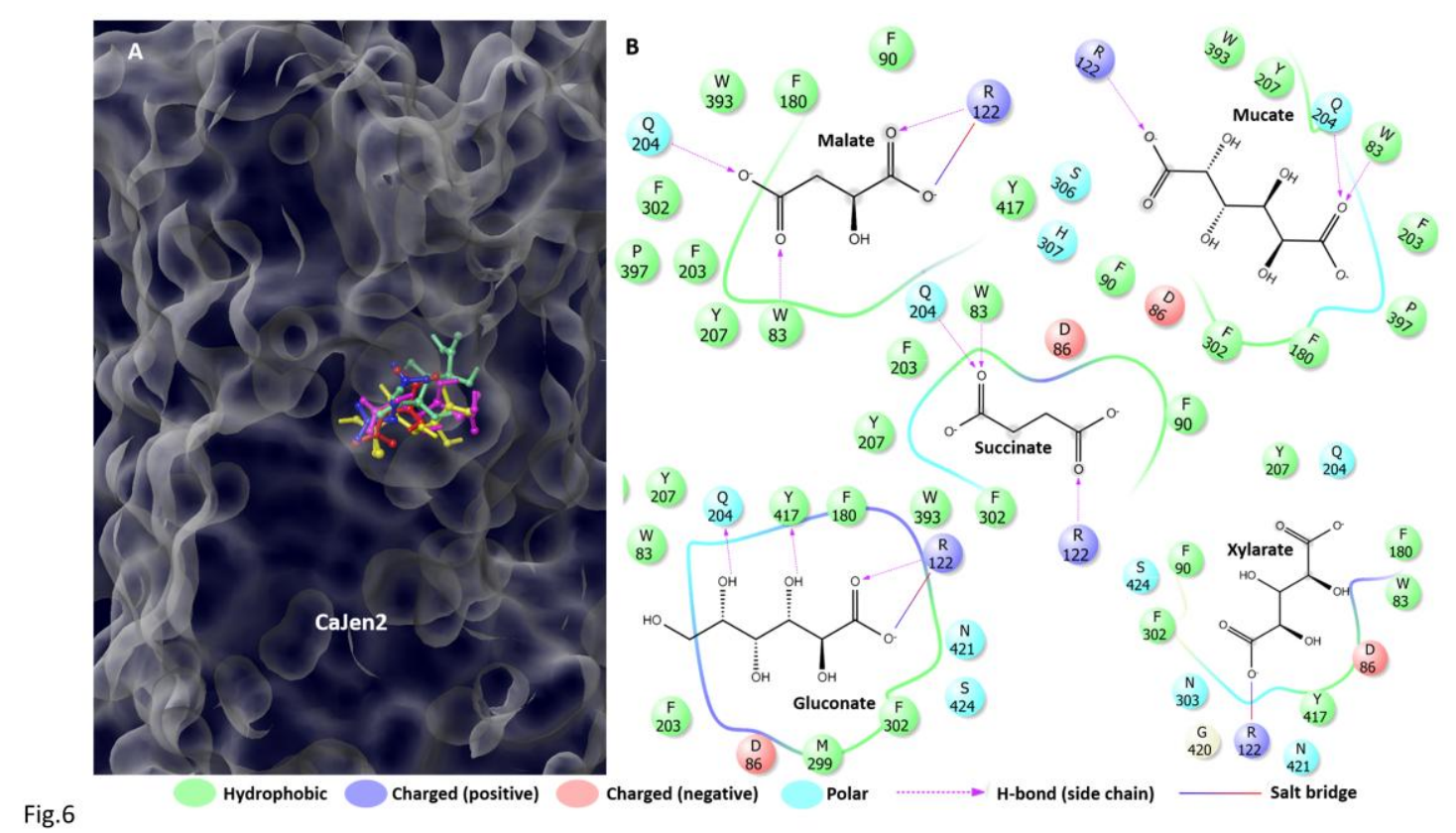

Figure 6. Molecular docking of CaJen2 with malate, succinate, gluconate, mucate and xylarate. A) Transversal view of CaJen2 protein surface with identification of the binding sites obtained in docking analysis. Ligand molecules are represented in their stick molecular structure coloured blue (succinate), red (malate), yellow (gluconate), purple (mucate) and green (xylarate). B) 2D view of the interactions between ligands (malate, succinate, gluconate, mucate and xylarate) and CaJen2 residues. The 3D CaJen2 structural model used in this study was modulated from the crystal structure of the GLUT3 transporter (PDB 4ZW9). 

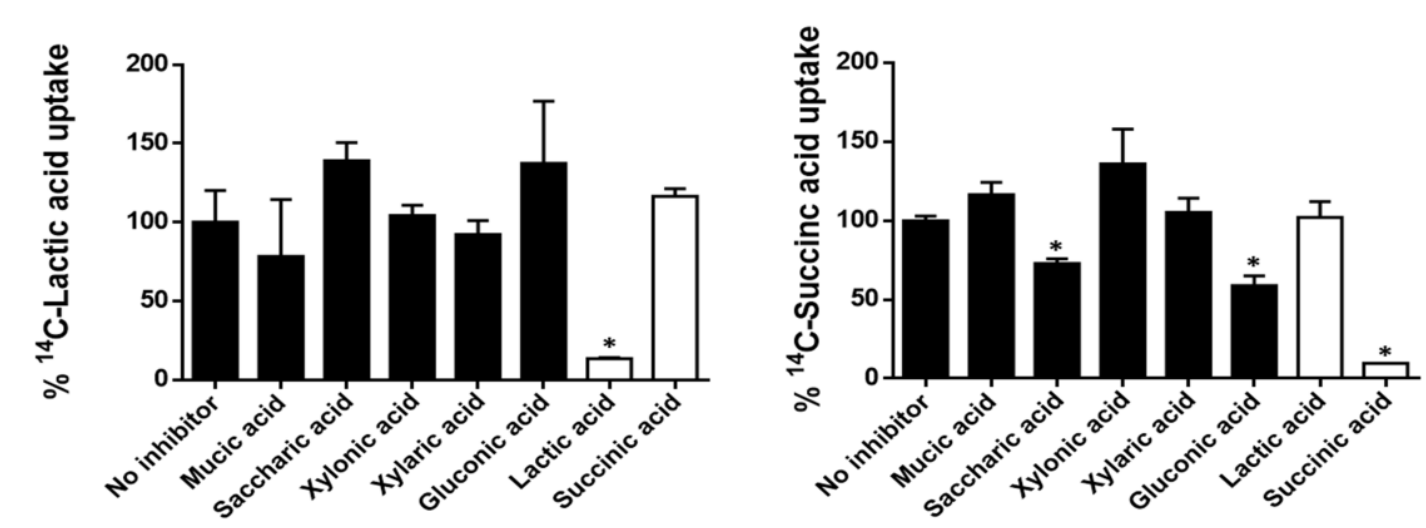

Fig.7

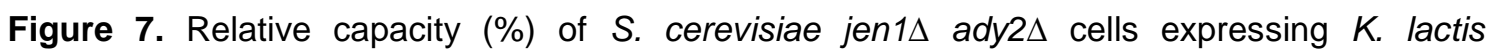
homologues (KIJen1 and KIJen2) to transport lactate and succinate in the absence and presence of mucic, saccharic, xylonic, xylaric and gluconic acid. A) ${ }^{14} \mathrm{C}$-lactic acid uptake $(60$ $\mu \mathrm{M})$ in cells transformed with pKIJen1. B) ${ }^{14} \mathrm{C}$-succinic acid $(20 \mu \mathrm{M})$ uptake in cells transformed with pKIJen2. The data shown are mean values of at least two independent experiments and the error bars represent the standard deviation. * Significant difference $(p \leq 0.05)$.

A

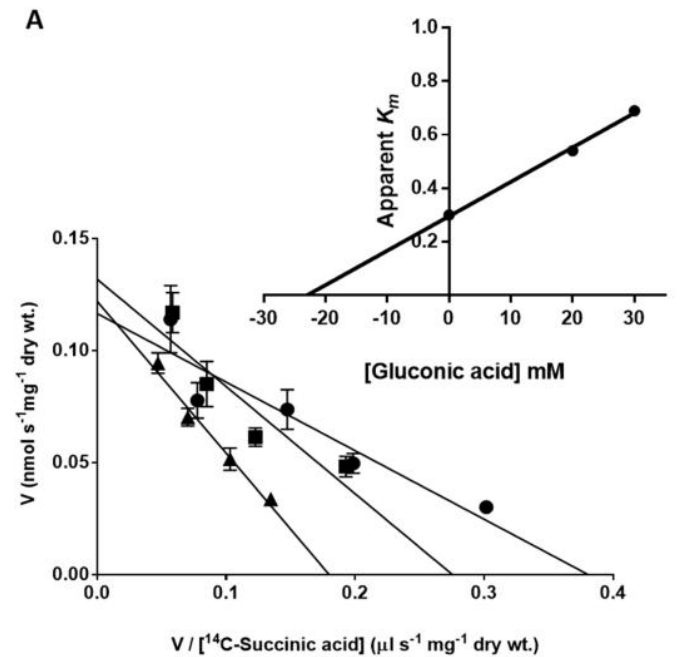

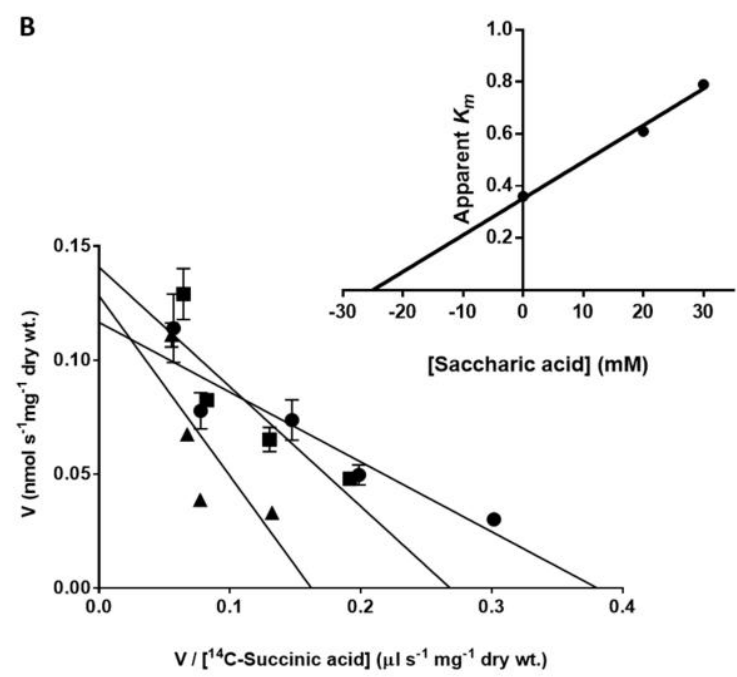

B

$\mathrm{V} / \mathrm{I}^{14} \mathrm{C}$-Succinic acid] $\left(\mu / \mathrm{s}^{-1} \mathrm{mg}^{-1} \mathrm{dry} \mathrm{wt}.\right)$

Fig.8

Figure 8. Eadie-Hoffstee plots of the initial uptake rates of ${ }^{14} \mathrm{C}$-succinic acid as a function of the acid concentration at $\mathrm{pH} 5.0,26^{\circ} \mathrm{C}$ in $S$. cerevisiae jen $1 \Delta$ ady2 $\Delta$ pKIJen2 cells in the presence of A) no inhibitor $(\bullet)$, gluconic acid $20 \mathrm{mM}(\boldsymbol{\bullet})$ and gluconic acid $30 \mathrm{mM}(\mathbf{\Delta})$; B) no inhibitor $(\bullet)$, saccharic acid $20 \mathrm{mM}(\boldsymbol{\square})$ and saccharic acid $30 \mathrm{mM}(\boldsymbol{\Delta})$. Insets: the apparent $K_{\mathrm{m}}$ of labelled succinic acid uptake against (A) gluconic and (B) saccharic acid concentration. The estimated $K_{i}$ is $K_{\mathrm{i}}$ is $22.7 \mathrm{mM}$ and $24.8 \mathrm{mM}$ for total gluconic and saccharic acid, respectively. The data shown are mean values of at least three independent experiments and the error bars represent the standard deviation. 

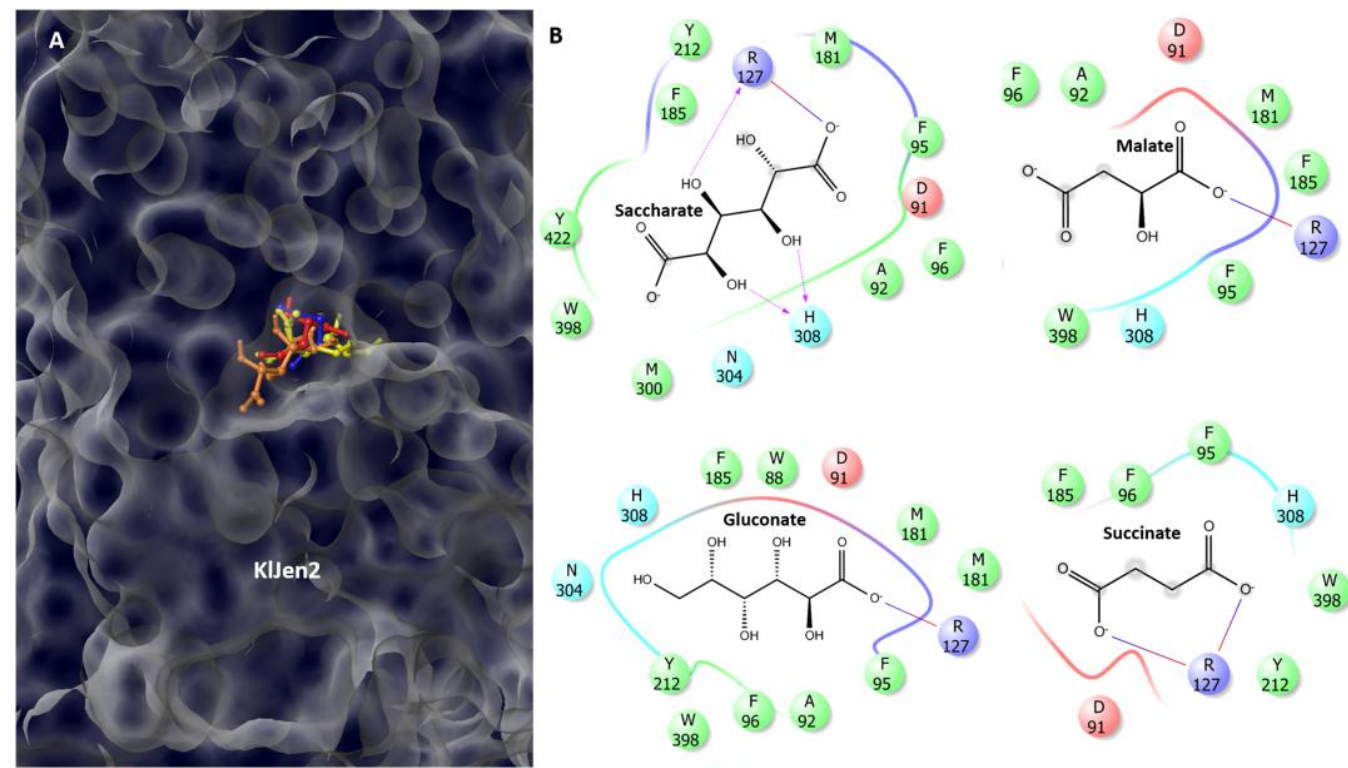

Fig.9
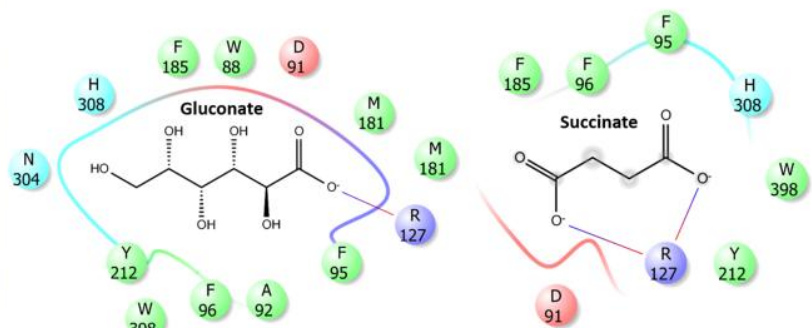

$\mathrm{W}$
398

Figure 9. Molecular docking of KIJen2 with malate, succinate, gluconate and saccharate. A) 3D transversal view of KIJen2 protein surface with identification of the ligands binding sites: succinate (blue), malate (red), gluconate (yellow) and saccharate (orange). B) 2D view of interactions between ligands (malate, succinate, gluconate and saccharate) and KIJen2 residues. The 3D KIJen2 structural model used in this study was obtained from the crystal structure of PiPT transporter (PDB 4J05). 
Table 1. Yeast species and strains used in this work.

\begin{tabular}{|c|c|c|}
\hline Strain & Genotype & Source or reference \\
\hline S. cerevisiae W303-1A & MATa ade2 leu2 his3 trp1 ura3 & $\begin{array}{l}\text { Thomas and Rothstein } \\
\text { (1989) }\end{array}$ \\
\hline S. cerevisiae jen $1 \Delta$ ady2 $\Delta$ & W303-1A; JEN1::KanMX4 ADY2::HphMX4 & Soares-Silva et al. (2007) \\
\hline S. cerevisiae jen1 $\Delta$ ady2 $\Delta$ p416GPD & jen1 $\Delta$ ady2 $\Delta$ transformed with p416GPD & Soares-Silva et al. (2007) \\
\hline S. cerevisiae jen1 $1 \Delta$ ady2 $\Delta$ pScJen1 & jen $1 \Delta$ ady2 $\Delta$ transformed with pScJen 1 & Soares-Silva et al. (2003) \\
\hline S. cerevisiae jen1 $\Delta$ ady2 $\Delta$ pScJen1-S271Q & jen1 $\Delta$ ady2 $\Delta$ transformed with pScJen1-S271Q & Soares-Silva et al. (2011) \\
\hline S. cerevisiae jen1 $\Delta$ ady2 $\Delta$ pScJen1-A272G & jen1 $\Delta$ ady2 $\Delta$ transformed with pScJen1-A272G & Soares-Silva et al. (2011) \\
\hline S. cerevisiae jen1 $1 \Delta$ ady2 $\Delta$ pScJen1-F270G & jen1 $\Delta$ ady2 $\Delta$ transformed with pScJen1-F270G & Soares-Silva et al. (2011) \\
\hline 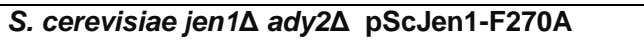 & jen1 $\Delta$ ady2 $\Delta$ transformed with pScJen1-F270A & Soares-Silva et al. (2011) \\
\hline $\begin{array}{l}\text { S. cerevisiae jen1 } \Delta \text { ady2 } \Delta \text { pScJen1- } \\
\text { F270Q/S271Q }\end{array}$ & $\begin{array}{l}\text { jen } 1 \Delta \text { ady2 } 2 \text { transformed with pScJen1- } \\
\text { F270Q/S271Q }\end{array}$ & Soares-Silva et al. (2011) \\
\hline S. cerevisiae jen1 $\Delta$ ady2 $\Delta$ pScJen1-Q498A & jen $1 \Delta$ ady2 $\Delta$ transformed with pScJen1-Q498A & Soares-Silva et al. (2011) \\
\hline S. cerevisiae jen1 $\Delta$ ady2 $\Delta$ pScJen1-Y284Q & jen1 $\Delta$ ady2 $\Delta$ transformed with $\mathrm{pScJen1-Y284Q}$ & Soares-Silva et al. (2011) \\
\hline S. cerevisiae jen1 $\Delta$ ady2 $\Delta$ pScJen1-Y284A & jen1 $\Delta$ ady2 $\Delta$ transformed with pScJen1-Y284A & Soares-Silva et al. (2011) \\
\hline S. cerevisiae jen1 $\Delta$ ady2 $\Delta$ pScJen1-Q386A & jen1 $\Delta$ ady2 $\Delta$ transformed with pScJen1-Q386A & Soares-Silva et al. (2007) \\
\hline $\begin{array}{l}\text { S. cerevisiae jen } 1 \Delta \text { ady2 } \Delta \text { jen } 1 \Delta \text { ady2 } \Delta \\
\text { pCaJen } 1\end{array}$ & jen $1 \Delta$ ady2 $\Delta$ transformed with pCaJen1 & Soares-Silva et al. (2004) \\
\hline $\begin{array}{l}\text { S. cerevisiae jen } 1 \Delta \text { ady } 2 \Delta \text { jen } 1 \Delta \text { ady } 2 \Delta \\
\text { pCaJen2 }\end{array}$ & jen $1 \Delta$ ady2 $\Delta$ transformed with pCaJen2 & Vieira et al. (2009) \\
\hline S. cerevisiae jen $1 \Delta$ ady2 $\Delta$ jen $1 \Delta$ ady $2 \Delta$ pKIJen 1 & jen1 $\Delta$ ady2 $\Delta$ transformed with pKIJen1 & Lodi et al. (2004) \\
\hline S. cerevisiae jen $1 \Delta$ ady2 $\Delta$ jen $1 \Delta$ ady $2 \Delta$ pKIJen 2 & jen $1 \Delta$ ady2 $\Delta$ transformed with pKIJen2 & Lodi et al. (2004) \\
\hline Candida albicans RM1000 & ura3::imm434/ura3::imm434, his1::hisG/his1::hisG & Negredo et al. (1997) \\
\hline Kluyveromyces lactis & Type strain CBS 2359 & CBS 2359 \\
\hline Yarrowia lipolytica & Type strain ISA 1718 & ISA 1718 \\
\hline Debaryomyces hansenii & Type strain CBS 767 & CBS 767 \\
\hline
\end{tabular}


Table 2. Plasmids used in this work.

\begin{tabular}{|l|l|l|}
\hline Plasmids & Characteristics & Source/reference \\
\hline p416GPD & Glyceraldehyde-3-phosphate dehydrogenase (GPD) promoter & $\begin{array}{l}\text { Mumberg, Muller and Funk } \\
\text { (1995) }\end{array}$ \\
\hline pScJen1 & ScJen1 cloned in p416 under the control of GPD promoter & Soares-Silva et al. (2003) \\
\hline pScJen1-S271Q & ScJen1-S271Q cloned in p416 under the control of GPD promoter & Soares-Silva et al. (2011) \\
\hline pScJen1-A272G & ScJen1-A271G cloned in p416 under the control of GPD promoter & Soares-Silva et al. (2011) \\
\hline pScJen1-F270G & ScJen1-F270G cloned in p416 under the control of GPD promoter & Soares-Silva et al. (2011) \\
\hline pScJen1-F270A & ScJen1-F270A cloned in p416 under the control of GPD promoter & Soares-Silva et al. (2011) \\
\hline pScJen1-F270Q/S271Q & ScJen1-F270Q/S271Q cloned in p416 under the control of GPD & Soares-Silva et al. (2011) \\
promoter
\end{tabular}

